\title{
High-Frequency Current Transformer Design and Implementation Considerations for Wideband Partial Discharge Applications
}

\author{
Joni V. Klüss, Member, IEEE, Alf-Peter Elg, Member, IEEE, and Claes Wingqvist
}

\begin{abstract}
High-frequency current transformers are popular noninvasive inductive wideband sensors. Despite simplicity in design and operational principle, implementation of such sensors for partial discharge applications requires careful consideration, particularly in the higher frequency range where traveling wave attenuation and distortion is relevant. First, the role of design variables, including core materials, winding design, and shielding practices on sensor sensitivity and frequency characteristics (transfer impedance) are presented. Next, the suitability of the constructed sensors for partial discharge applications is assessed. The designed wideband sensors are suitable for laboratory applications with standard measurement circuits and controlled conditions. The low-level magnitude and frequency spectrum of the discharge pulses hinders signal integrity in relation to the placement of the sensors within the measurement circuit, signal amplification, and pulse repetition rate (pulse resolution). To enable most stringent detection levels under 1 pC, efforts are needed in distortionless amplifier design and interference mitigation.
\end{abstract}

Index Terms - current transformers, frequency-domain analysis, high-voltage techniques, partial discharge measurement, time-domain analysis.

\section{INTRODUCTION}

$\mathrm{P}$ ARTIAL discharges (PD) are of great interest to widely ranging research and application themes. In general, one strives to collect representative data of the PD event that enables reliable interpretation of the system, device, or material status. However, the physical PD occurrence cannot be directly measured without altering the event. As illustrated in Fig. 1, PD manifests itself in numerous forms. Charge migration during the discharge event can produce electrical current pulses that can be observed using appropriate instruments; resulting electromagnetic radiation can be detected with appropriate antennas; pressure waves can be recorded using acoustic sensors; generated heat can be picked up by thermal imaging; and so on. One measurement technique may not necessarily detect all forms of PD - some sensors are more effective than others in observing specific PD phenomena. All of these different techniques are correlated to the PD phenomena, but

Manuscript submitted for review 08/19/2020. This work was supported by the Swedish Governmental Agency for Innovation Systems and by the project 19ENG02 (Metrology for future energy transmission) from the EMPIR program co-financed by the Participating States and from the European Union's Horizon 2020 research and innovation program. are impacted by a range of influential variables including the coupling device (sensor response), measurement circuit (acquisition/sampling unit, data transfer components), signal post processing and filtering, and prevailing ambient conditions during measurements, just to name a few. Ideally, a system is completely PD free. However, some degree of imperfections always exists, which may eventually trigger PD events. Unfortunately, outside controlled laboratory test conditions, the onset, the source, the location, and most importantly, the severity of PD is unknown in advance. In worst case scenarios, improper measurement techniques may be blind to PD events, making a system appear to be PD free. Even, upon detection of $\mathrm{PD}$, correlating the registered data to the severity of the event is challenging. i.e., what is considered acceptable levels of PD.

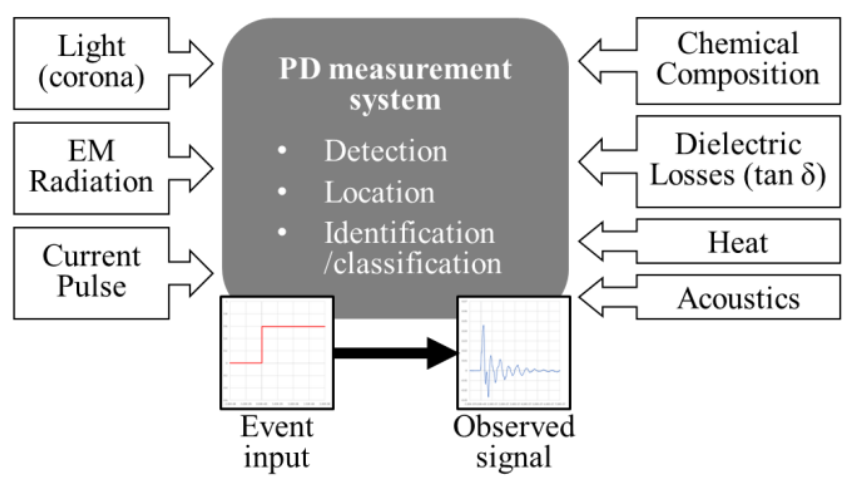

Fig. 1. PD information can be derived from alternative sources related to the event. Measurement systems can have widely varying responses, altering the signals observed by the end-user.

This paper is an extension of the CPEM 2020 proceedings paper [1] investigating challenges associated with measuring PD using wideband high-frequency current transformers (HFCTs). Such sensors are simple in design and can be manufactured with relatively minimal resources. Installation and utilization is also straightforward as split-core solutions allow for clamping around conductors without the need for service interruptions, disassembly, and tools. For PD applications, in addition to the ability to detect the small rapid

J. V. Klüss and A-P. Elg, are with the High Voltage unit at RISE Research Institutes of Sweden, SE-50115 Borås, Sweden (e-mail: joni.kluss@ ri.se).

C. Wingqvist is with the Temperature and Electrical Primary Metrology unit at RISE Research Institutes of Sweden, SE-50115 Borås, Sweden (e-mail: claes.wingqvist@ri.se). 
event, key functions for a sensor also include the ability to provide information on the defect type and estimate its severity using derived parameters from the recorded signal. This paper demonstrates the validity and empirical limitations of existing conventional practices and techniques extending beyond standard specifications to help shape the direction for future development of wideband PD sensors.

\section{PD MEASUREMENT PRINCIPLES}

Conventional AC measurement techniques for PD (Phase Resolved Partial Discharge Analysis, PRPDA) are wellestablished and described in IEC 60270 [2]. Fundamental quantities related to magnitude and phase angle can be derived from measurements and analyzed for fault discrimination and finger printing. The PD current pulse $i(t)$, expressed as a double exponential function, provides charge $q$ as

$$
q=\int_{0}^{\infty} i(t) d t
$$

IEC 60270 defines ranges for wideband instruments with lower frequency limits $30 \mathrm{kHz} \leq f_{1} \leq 100 \mathrm{kHz}$, upper limits $f_{2} \leq$ $1 \mathrm{MHz}$, and bandwidth $100 \mathrm{kHz} \leq \Delta f \leq 900 \mathrm{kHz}$. Such wideband instruments observe well-damped oscillatory responses enabling the determination of apparent charge and the polarity of the pulse. Pulse resolution (shortest time interval between two consecutive pulses) is typically 5-20 $\mu$ s. Narrowband instruments (bandwidth $9 \mathrm{kHz} \leq \Delta f \leq 30 \mathrm{kHz}$, and midband frequency $50 \mathrm{kHz} \leq f_{\mathrm{m}} \leq 1 \mathrm{MHz}$ ) produce oscillatory responses with envelope values proportional to apparent charge, but independent of the polarity. Pulse resolution is typically longer than $80 \mu \mathrm{s}$.

Despite varying instrument responses, within the confines of IEC 60270, the concept of "apparent charge" and "calibration" is valid. Calibration is performed to determine the scale factor $k$ for the measurement of the apparent charge by injecting rapid current pulses of known charge magnitude into the terminals of the test object. A PD measuring system working in this "low" frequency range detects the constant part of the PD pulse frequency response. "Since the upper cut-off frequency of the detection bandpass is significantly lower than the upper cut-off frequency of the pulse frequency response, the detected PD pulses are directly proportional to the apparent charge of the PD current pulse" (quasi-integration) [3]. When the test object can no longer be represented as a simple lumped capacitance, and frequencies extend into the HF-UHF regime, the validity of calibration (correlation to apparent charge) becomes void. This occurs for larger complex devices such as transformers, rotating machines, long cables, and GIS which exhibit transmission line characteristics [4]. Here, only a portion of the PD energy arrives at the sensors, whereupon PD magnitude becomes a relative measure of PD activity and "calibration" is replaced by "normalization". A direct relation between voltage $(\mathrm{mV})$ and charge $(\mathrm{nC})$ is questionable as the observed signals originating from an unknown location are distorted by pulse propagation phenomena (reflections, resonance, cross-coupling, etc.) [3].

A higher low-frequency cutoff threshold increases the probability of neglecting PD signals distant from the sensor since high frequency components of the signal are considerably attenuated and may become completely undetectable [3]. Nevertheless, the push for wideband operation extending to higher upper frequency ranges is motivated by interference rejection and suppression principles. Lower frequency ranges are subject to more noise and disturbances (a larger scope of signal sources, including undesired signals) [5]. This is particularly relevant when the voltage supply itself produces rapid excitations, e.g., converter fed motor switching with repetitive short rise time voltage impulses. Furthermore, DC applications, which lack phase information enabling conventional PD quantity derivation, implement pulse shape analysis based on, e.g., rise-time, decay time, and pulse-width [3]. Waveform parameters derived from individual PD pulses can be used for the characterization of different defects using clustering techniques for separation of multiple sources (defects) and noise [6][7][8][9][10][11][12]. Pulse shape analysis, thus, requires high resolution details and clear understanding of the sensor characteristics with which said signals are observed.

\section{HFCT DESIGN}

Current transformers are inductive couplers widely used as non-intrusive measurement devices converting time-varying current into a voltage (or current) signal scaled by the number of turns in the transformer. Power-frequency applications generally implement grain-oriented silicon-iron cores while HFCTs utilize metallic oxide materials (ferrites) [13]. HFCTs are often installed on the ground connection of a device where a current flowing along the conductor through the HFCT (single turn primary) induces a voltage measured across a resistive load. This induced voltage in the secondary is proportional to the rate of change of current in the primary [15]. Although simple in operational principle, design characteristics play a crucial role in the suitability of the HFCT for partial discharge applications. According to [17] and [26], sensors with flat and wideband frequency responses allow for detection of pulse shape-related features but have a tradeoff in reduced gain. For circumstances where accurate pulse shape is not a priority, sensitivity can be improved by designing a higher gain HFCT sensor with a non-flat ("peaky") frequency response. Here one must acknowledge that such a sensor distorts the pulse shape since different frequency components are amplified and phase shifted by different levels.

\section{A. Core Material and Winding Design}

Soft ferrite cores such as manganese-zinc (MnZn) and nickel-zinc $(\mathrm{NiZn})$ are effective couplers between electric current and magnetic flux [14]. Sensitivity of the sensor is significantly improved using such materials. However, ferromagnetic cores introduce nonlinearity to the transfer function (dependent on e.g., frequency, temperature, flux density) [15]. Initial investigations assess variability within the N30-type MnZn cores shown in Table 1. Relatively similar physical dimensions were selected to allow for mounting within same sized enclosures. 
TABLE I

CHARACTERISTICS OF N30-TYPE SENSORS

\begin{tabular}{lcccc}
\hline \hline & HFCT & HFCT & HFCT B & HFCT \\
& $\mathrm{A}$ & $\mathrm{B}$ & Double core & $\mathrm{C}$ \\
\hline Material & $\mathrm{N} 30$ & $\mathrm{~N} 30$ & $\mathrm{~N} 30$ & $\mathrm{~N} 30$ \\
Inductance $[\mu \mathrm{H}]$ & 8.2 & 5.2 & 10.5 & 5.8 \\
Relative permeability $\mu_{\mathrm{r}}$ & 4300 & 4300 & 4300 & 4300 \\
\hline \hline
\end{tabular}

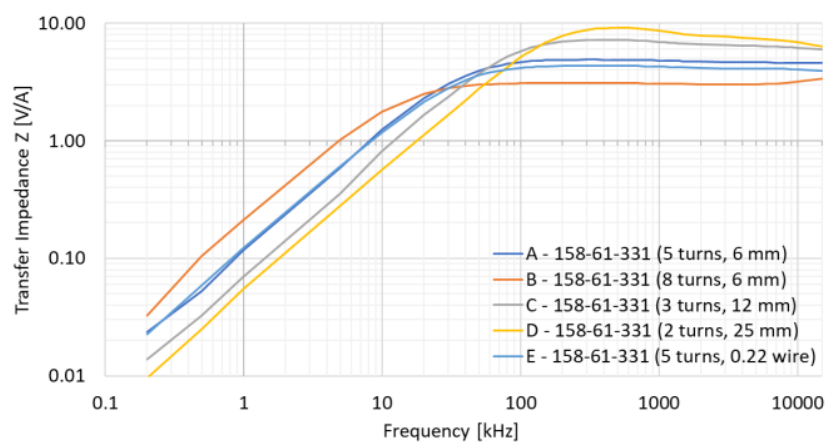

Fig. 2. Measured transfer impedance of HFCT A variations.

Winding type and number of turns was varied for "HFCT A" designs - copper adhesive tape of widths $6 \mathrm{~mm}, 12 \mathrm{~mm}$, and 25 $\mathrm{mm}$ was compared with $0.22 \mathrm{~mm}^{2}$ covered conductor winding. HFCT applications typically implement low number of turns to extend the effective operating range (upper cutoff frequency) to higher values. Since primary turns are generally fixed as $N_{\mathrm{p}}=1$ (ground conductor), increasing the number of secondary turns $N_{\mathrm{s}}$ shifts operation to lower frequencies and reduces sensitivity as apparent in Fig. 2. Significant variation between wire and strip (tape) windings was not observed. Based on the flatness of the transfer impedance for $N_{\mathrm{s}}=5$, this design was selected for further development.

\section{B. Shielding - Aperture Design}

An aperture (slit in the sensor enclosure) is said to improve coupling between the current carrying conductor and the winding [16] and prevents the formation of a short circuit turn around the core. Several designs were investigated (Fig. 3). For apertures located within the inner diameter of the ferrite core, aperture size influences the sensitivity of the sensor (design B) - larger aperture correlates to higher gain. As expected, highest sensitivity is achieved without any shielding between the current carrying conductor and the enclosure (design A). Interestingly, design $\mathrm{C}$ exhibits near identical characteristics to design A as evident by the overlaid plots in Fig. 4. Allowing for circulating currents using two-point grounding (short-circuit turn around core) is effective in hindering coupling up to $1 \mathrm{MHz}$ whereupon sensitivity returns to similar values as with the other designs.

\section{Bandwidth}

Transfer impedance and low-frequency cutoff values were determined by measuring the output voltage of the HFCT (NI PXIe 5164, $400 \mathrm{MHz}, 1 \mathrm{GS} / \mathrm{s}$ ) and dividing by the measured current (Tektronix TDS3054B $500 \mathrm{MHz}$ digitizer; TCP202 $50 \mathrm{MHz}$ current probe). Sinusoidal current of varying frequency up to $15 \mathrm{MHz}$ was supplied by a HP 33120A signal generator to a $50 \Omega$ termination via short twisted conductors

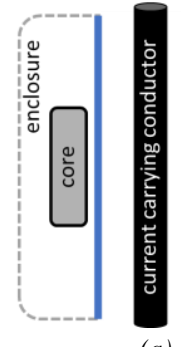

(a)

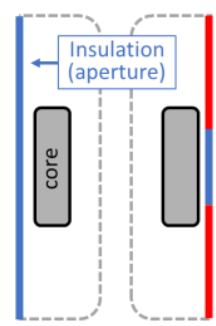

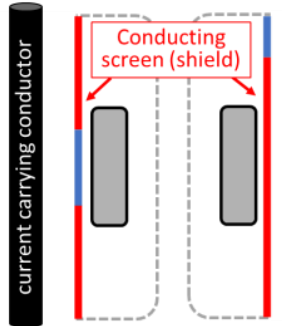

(b)

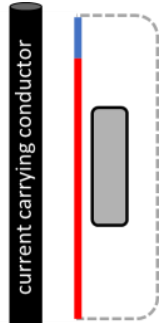

(c)
Fig. 3. Aperture designs: (a) full aperture (no screen); (b) aperture located at ferrite core height; (c) aperture above core location.

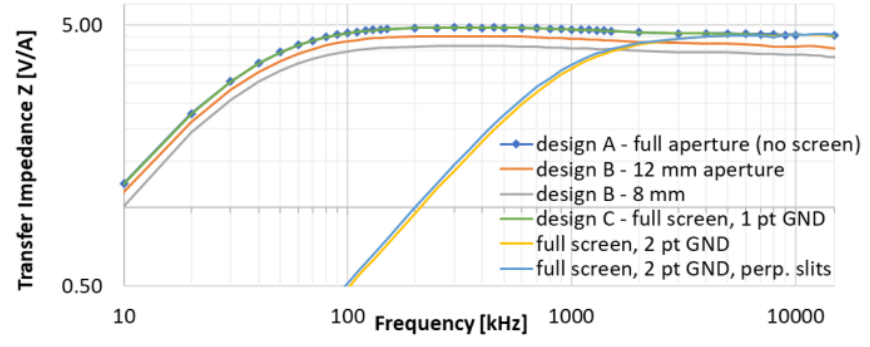

Fig. 4. Influence of aperture design on transfer impedance of HFCT A.

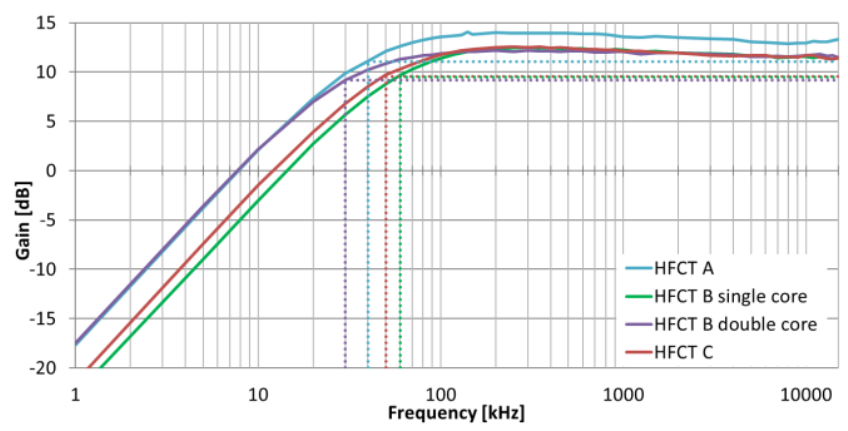

Fig. 5. Low-frequency cutoff for N30-type cores.

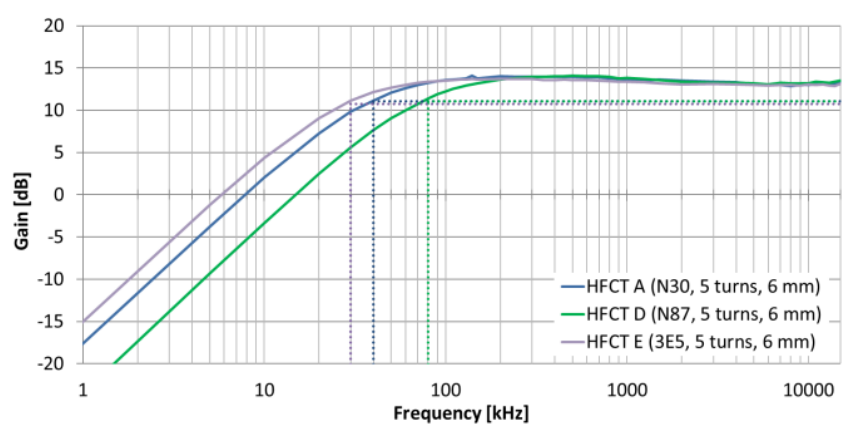

Fig. 6. Low-frequency cutoff for core types N30, N87, and 3E5.

(one passing though the HFCT and the other for return current). Results for N30-type cores are shown in Fig. 5. HFCT B and C have slightly lower sensitivity compared to HFCT A. Increasing the number of cores (HFCT B double core) to increase inductance does not result in larger gain, but is noticeable as a lower cutoff frequency.

HFCT A was selected as a reference for N30-type cores and compared to materials with varying permeability and inductance (refer to Table II). As evident in Fig. 6, all three sensors exhibit similar gain magnitudes (same number of turns, same enclosure design) while core selection is evident in the frequency domain - higher permeability and inductance extends the frequency range to lower values (and vice versa). 


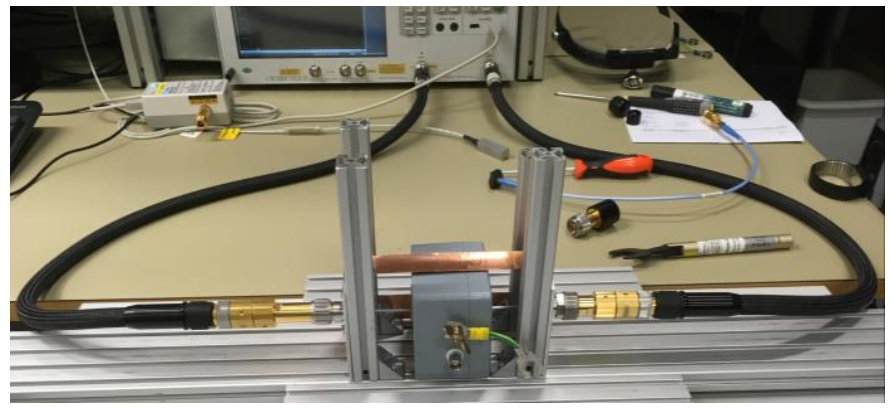

Fig. 7. VNA test assembly for S-parameter measurements consisting of bare conductor between $\mathrm{N}$-connectors passing through HFCT and multiple copper strips used as ground paths.

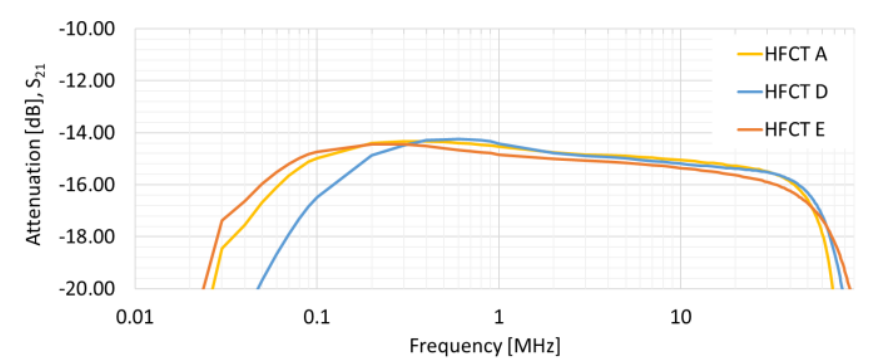

Fig. 8. Measured $\mathrm{S}_{21}$ for HFCT A, D, and E (accounting for attenuation caused by test assembly).

TABLE II

CHARACTERISTICS OF DEVELOPED HFCTS

\begin{tabular}{|c|c|c|c|}
\hline & HFCT A & HFCT D & HFCT E \\
\hline Material (MnZn) & N30 & N87 & $3 \mathrm{E} 5$ \\
\hline Core inductance $L$ & 8.2 & 4.2 & 11.1 \\
\hline Relative permeability $\mu_{\mathrm{r}}$ & 4300 & 2200 & 8500 \\
\hline Secondary turns $N_{\mathrm{s}}$ & 5 & 5 & 5 \\
\hline Inner diameter [mm] & 28.5 & 28.5 & 26.0 \\
\hline Outer diameter [mm] & 51.8 & 51.8 & 42.0 \\
\hline Length $[\mathrm{mm}]$ & 21.3 & 21.3 & 18.0 \\
\hline Load resistance $R[\Omega]$ & 50 & 50 & 50 \\
\hline $\begin{array}{l}\text { Measured secondary coil } \\
\text { parasitic capacitance } C_{2}[\mathrm{pF}]\end{array}$ & 41 & 15 & 17 \\
\hline $\begin{array}{l}\text { Measured secondary coil } \\
\text { inductance } L_{2}[\mu \mathrm{H}]\end{array}$ & 207 & 108 & 260 \\
\hline $\begin{array}{l}\text { Measured mutual inductance } \\
M \text { (from LF slope) }[\mu \mathrm{H}]\end{array}$ & 20.7 & 10.8 & 27.7 \\
\hline \multirow{3}{*}{$\begin{array}{l}\text { Measured transfer impedance } \\
\text { [V/A] } \\
\text { Measured gain (S-parameters) } \\
\text { Calculated gain } \mathrm{H} \text { [V/A] } \\
\left(=R M / L_{2}\right)\end{array}$} & 5.06 & 5.06 & 4.85 \\
\hline & 5.20 & 5.15 & 5.27 \\
\hline & 5.00 & 5.01 & 5.33 \\
\hline \multirow{4}{*}{$\begin{array}{l}\text { Measured lower limit } f_{\text {lower, }-3 \mathrm{~dB}} \\
\text { Calculated lower limit } f_{\text {lower, }-3 \mathrm{~dB}} \\
\left(=R / 2 \pi L_{2}\right) \\
\text { Measured upper limit } f_{\text {upper, }-3 \mathrm{~dB}} \\
\text { Calculated upper limit } f_{\text {upper, }-3 \mathrm{~dB}} \\
(=1 / 2 \pi R C)\end{array}$} & $40 \mathrm{kHz}$ & $80 \mathrm{kHz}$ & $30 \mathrm{kHz}$ \\
\hline & $38 \mathrm{kHz}$ & $74 \mathrm{kHz}$ & $30 \mathrm{kHz}$ \\
\hline & $57 \mathrm{MHz}$ & $61 \mathrm{MHz}$ & $65 \mathrm{MHz}$ \\
\hline & $44 \mathrm{MHz}$ & $85 \mathrm{MHz}$ & $74 \mathrm{MHz}$ \\
\hline
\end{tabular}

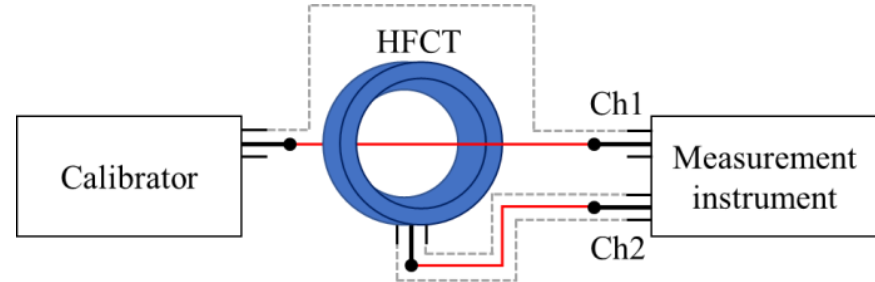

Fig. 9. HFCT measuring stable repetitive calibrator pulse supplied directly to measuring instrument.
The aforementioned technique for defining transfer impedance is limited to $15 \mathrm{MHz}$ by the signal generator. To obtain upper frequency values, an Agilent Technologies E5061B, $5 \mathrm{~Hz}-3 \mathrm{GHz}$, vector network analyzer (VNA) was utilized to measure S-parameters for HFCT A, D, and E (Fig. 7). Low frequency limitations of the VNA electronic calibrator module (Agilent N4690-60004, $300 \mathrm{kHz}-18 \mathrm{GHz}$ ), along with test assembly grounding contacts requiring disassembly and reconnection for mounting each HFCT, caused instability in measured data which was observed as a fluctuating offset or overshoot of several $\mathrm{dB}$ in measured values (i.e., gain) in the frequency range of $1 \mathrm{MHz}$. Nevertheless, lower cutoff frequencies based on measured $S_{21}$ parameters coincided well those obtained from the transfer impedance measurements. Sparameter values above $1 \mathrm{MHz}$ were stable. The influence (attenuation) of the test assembly without the HFCT was also measured and was removed from the recorded S-parameters which results in slightly reduced upper cutoff frequency values. The frequency-amplitude spectrum of the three HFCTs is presented in Fig. 8 and relevant properties summarized in Table II. Reliably measuring parasitic capacitance is difficult and thus causes discrepancy in calculated theoretical values for the upper cut-off frequencies compared to measured empirical values.

\section{PD APPLICATIONS}

In general, PD impulses are characterized as short risetime narrow pulses with varying frequency spectrums, mostly under tens to hundreds of $\mathrm{MHz}$, but some extending into the UHFregime (dependent on defect type, test object, applied voltage stress, measurement and acquisition techniques, etc.) [8][13] [17][18][19][20][21][22][23][24][25]. Comparability of research data is limited and a product of each measurement setup and associated transfer functions. Even for a fixed test assembly, PD data is stochastic by nature. To enable characterization of the developed HFCT designs, a repeatable stable PD source was needed. An Omicron CAL 542 calibrator $(1-100 \mathrm{pC})$ was used to supply a repetitive, c. 4 ns risetime, pulse directly to a NI PXIe 5164 digitizer via short conductors as illustrated in Fig. 9.

For $100 \mathrm{pC}$ calibrator pulses, HFCT A consistently observed slightly slower waveforms while HFCT D and E responded with very similar characteristics (Fig. 10). The recorded calibrator signal's highest significant frequency component is at $37-41 \mathrm{MHz}$, within the bandwidths of the HFCTs. All sensors detect rather similar peak values, with the ratio between calibrator peak value and HFCT values on average 6.3 (standard deviation $s=4.1 \%), 6.1(s=4.3 \%), 6.3(s=2.0 \%)$ within the entire $1-100 \mathrm{pC}$ calibrator range for HFCT A, D, and $\mathrm{E}$ respectively. Signal to noise ratio SNR worsens for the smaller calibrator injections $1-5 \mathrm{pC}$ with zero-level and front oscillations hindering the accurate determination of peak values and risetime as demonstrated in Fig. 11 for $1 \mathrm{pC}$ charge injections.

\section{A. Impact of Amplification}

Injecting such calibrator pulses into circuits including resistive, capacitive, and inductive components, external 


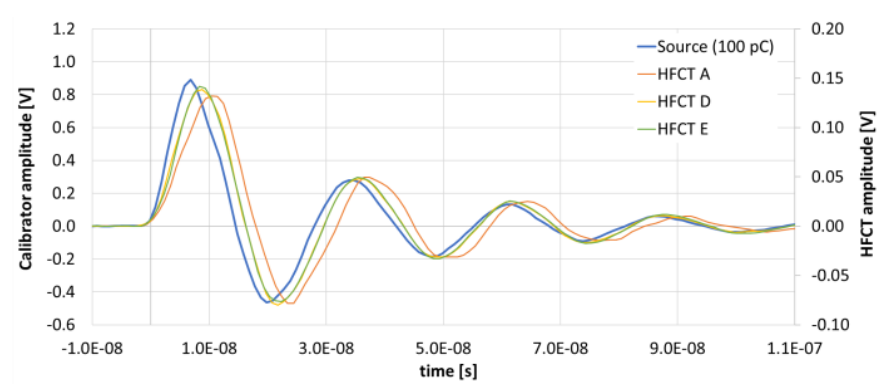

Fig. 10. Response of HFCT sensors to $100 \mathrm{pC}$ calibrator pulses.

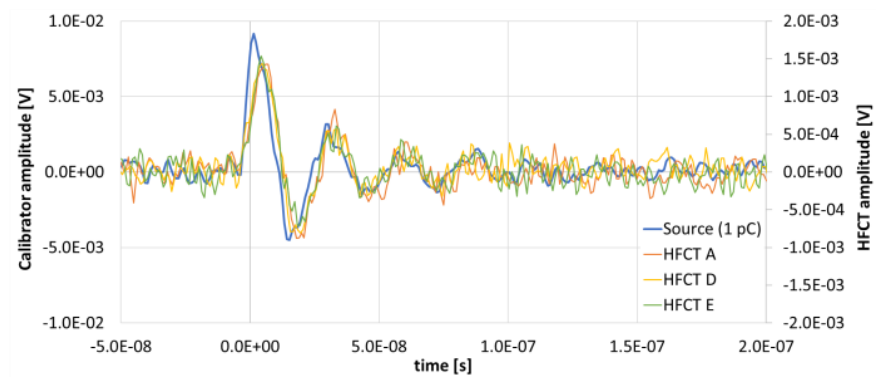

Fig. 11. Response of HFCT sensors to $1 \mathrm{pC}$ calibrator pulses.

interference sources, and other "real-world" nonidealities, further alters the characteristics of the signal and can mask relevant features in excessive background noise. In addition to interference mitigation techniques, amplification may be needed to meet most stringent acceptance criteria.

A $1 \mathrm{pC}$ calibration pulse was injected across a $0.5 \mathrm{pF}$ dummy test object in a conventional PD test setup. The HFCT signal was fed through a Tektronix AM502 differential amplifier (10 $\mathrm{kHz}-1 \mathrm{MHz}$ bandwidth) to the NI PXIe 5164 digitizer. Amplifier gain was varied to determine the linearity of the amplification range. As evident in Fig. 12, the slower response of the amplifier considerably alters the HFCT response. Nevertheless, waveform characteristics of the amplified signal remain constant for $100-5000$ gain factors. The amplification of zero level noise for the highest gain settings results in some distortions, but peak detection of the amplified signal is considerably improved. However, the slower response has an impact on achievable pulse repetition rates. The amplified signal begins to distort the pulse train response at $250 \mathrm{kHz}$ while the unamplified HFCT signal is still distinguishable above 2 $\mathrm{MHz}$ repetition rates (Fig. 13).

Such superposition of subsequent pulses can result in misleading PD analysis as demonstrated in Fig. 14 when measuring Trichel pulses (negative corona). As applied ACvoltage is increased, the occurrence of Trichel pulses increases - greater number of discharges within the negative half-cycle visualized as a wider area of consistent amplitude pulses. The accumulation of signals at the extremities of the pulse distribution for $\mathrm{C} 2$ and $\mathrm{C} 3$ is not a physical phenomenon related to the PD process, but instead a consequence of signal superposition due to insufficient settling time between discharge pulses.

Pulse repetition rate has a significant impact on a key PD parameter -i.e., charge estimation. [26] applies two approaches for estimating charge, a "simplified HFCT model" and a "generic HFCT model" where both techniques implement

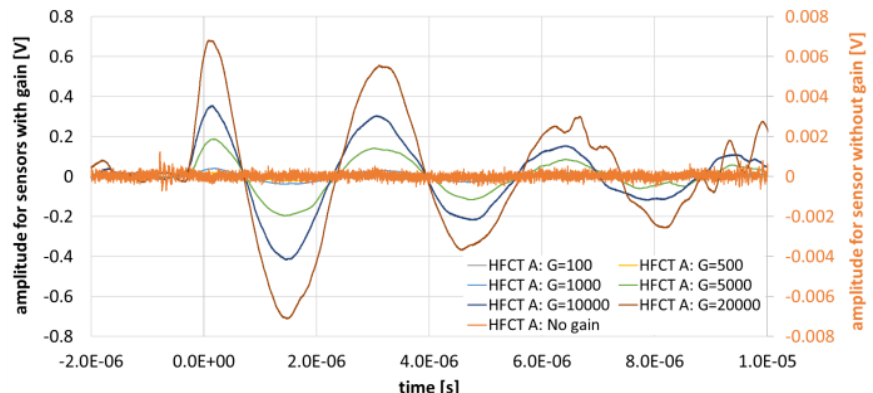

Fig. 12. Influence of differential amplifier on $1 \mathrm{pC}$ calibrator injection measured by HFCT.

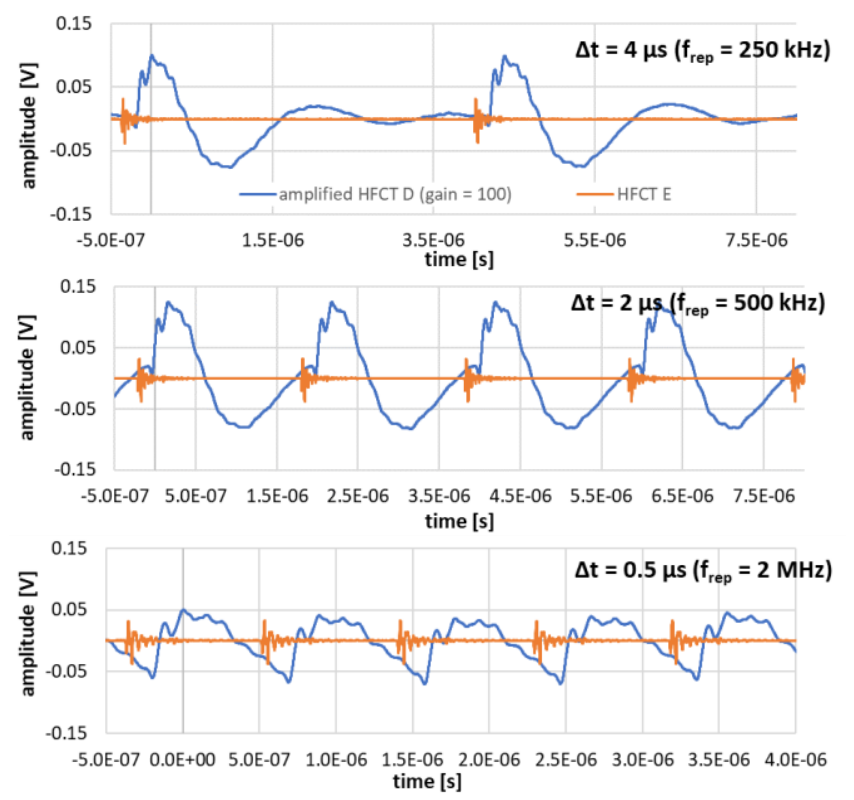

Fig. 13. Influence of pulse repetition rate. Repetitive pulses supplied by Philips PM 5712 pulse generator, risetime 4 ns, pulse width 120 ns.

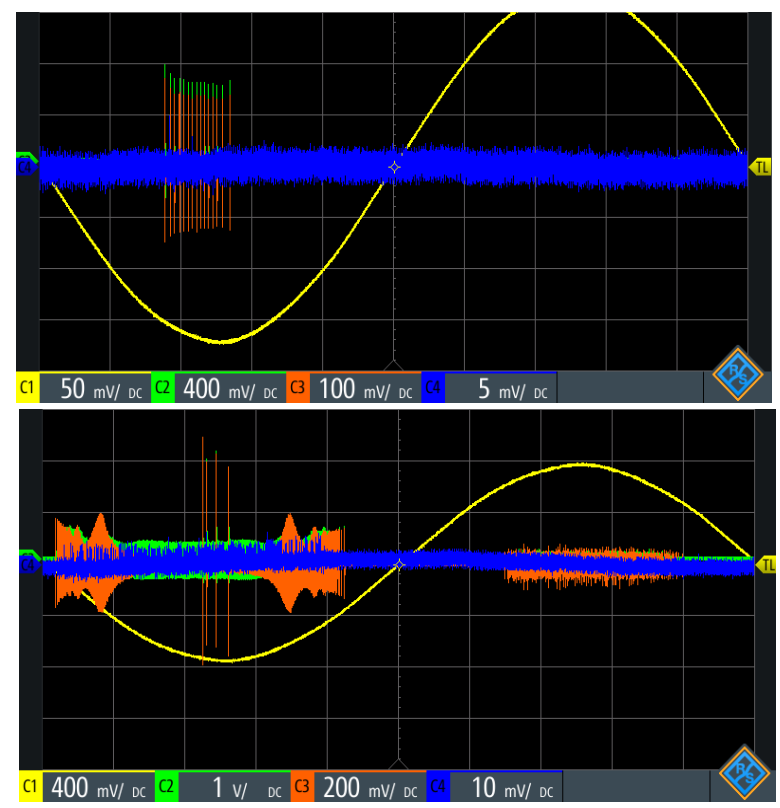

Fig. 14. Signal distortion caused by slow sensor response to repetitive discharge. $\mathrm{C} 1$ is applied voltage, slower $\mathrm{C} 2$ and $\mathrm{C} 3$ signals are obtained with IEC 60270 compliant coupling devices, and C4 is a shunt prototype with a fast response but poor sensitivity. Top - initial onset of Trichel pulses; bottom misrepresentation of phase resolved PD data due to poor pulse resolution of coupling devices. 
voltage proportionality to total charge in relation to integration time. The simplified model is given as,

$$
q \approx \frac{1}{H} \int_{t_{2}}^{t_{1}} u(t) d t
$$

Gain $H$ is calculated as $R M / L_{2}$ where $R$ is loading resistance and $L_{2}$ is secondary winding inductance. Mutual inductance $M$ is calculated from the linear slope of the HFCT transfer function in the low-frequency range. For the generic model,

$$
q \approx \frac{1}{M} \iint_{0}^{4 L_{2} / R} u(t) d t^{2}
$$

Integration time (approximated as $4 L_{2} / R$ ) is related to the poles of the sensor transfer function which defines lower and upper cutoff frequencies $\left(f_{\text {lower }}=R / 2 \pi L_{2}\right.$ and $f_{\text {upper }}=1 / 2 \pi R C$ ). Flat wideband sensors require longer integration times compared to those with peaky response. Fig. 13 demonstrated that sufficient deadtime is achieved to discriminate between subsequent pulse shapes. However, for charge estimation, pulse repletion rates faster than the integration time will include multiple signals and thereby result in erroneous values.

Shorter integration times are achieved by shifting the lower cutoff frequency (proportional to $R / L_{2}$ ) to higher values. This can be achieved with smaller $L_{2}$ by reducing the number of turns or alternatively selecting a core with smaller inductance $\left(L_{2}=\right.$ $L N^{2}$ ). Although a smaller core inductance $L$ corresponds to larger gain, sensor bandwidth is decreased. Increasing lower cutoff frequencies may be desirable for filtering disturbances but can in turn risk losing relevant PD information related to the event of interest. One of the fundamental design questions is thus related to the intended purpose of the sensor - pulse shape analysis or magnitude (charge) estimation.

\section{B. Impact of HFCT Placement}

Despite being nonintrusive and non-loading, the installation location of an HFCT impacts the observation of PD events. Influence of placement and grounding was investigated by varying configurations within the conventional IEC60270 circuit shown in Fig. 15. From the perspective of waveform integrity (pulse shape analysis), the dominant characteristic for HFCT applications intended for wideband PD pulse acquisition appears to be related to travelling wave propagation. In typical context, one often considers the transmission characteristics of travelling waves as they propagate from the source to the measurement point, e.g., time-difference-of-arrival (TDOA) analysis for underground cables, overhead lines, or GIS. For HFCT installations on the ground path of circuits, it is necessary to consider what happens to the signal after it passes the sensor location. I.e., in addition to transmission within the test object, the ground conductor, and in particular, the ground termination, need to also be considered part of the transmission path. Good grounding practices strive to minimize ground conductor lengths. Results suggest that the length of ground conductor between the test object and laboratory ground common point is not as critical as the position of the HFCT along said length of ground conductor (for PD applications).

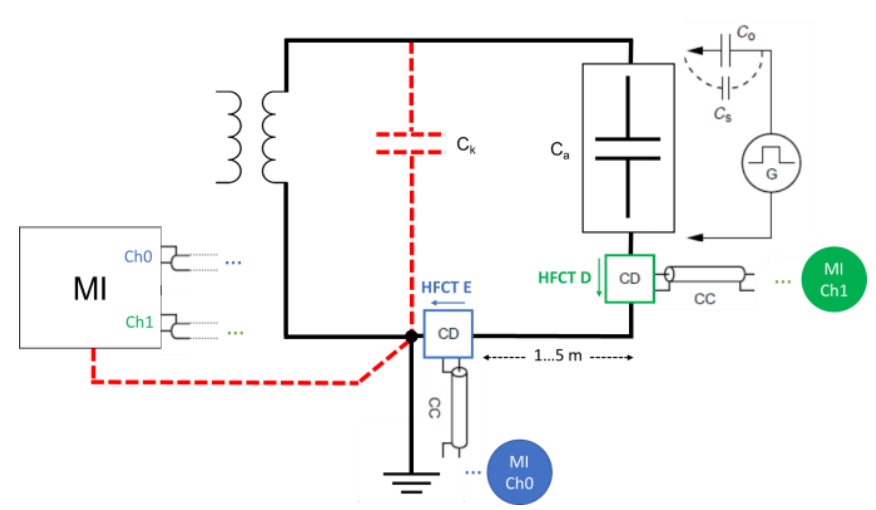

Fig. 15. Conventional PD circuit with varying HFCT location along the ground conductor of dummy test object $C_{\mathrm{a}}$ (parallel disc-electrode gap, c. $0.5 \mathrm{pF}$ ). The presence (or absence) of coupling capacitor $C_{\mathrm{k}}$ does not influence the performance of the HFCT.

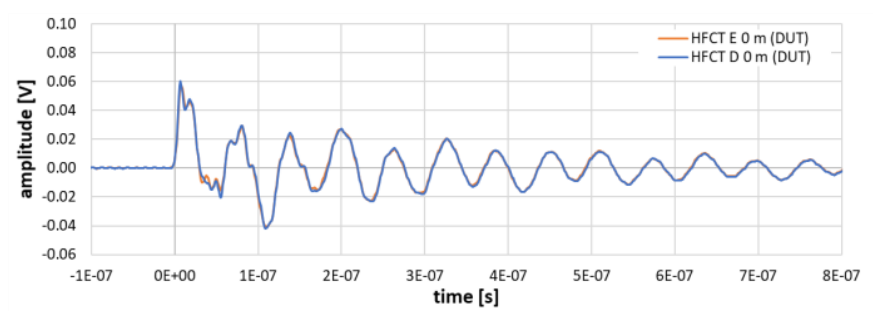

(a)

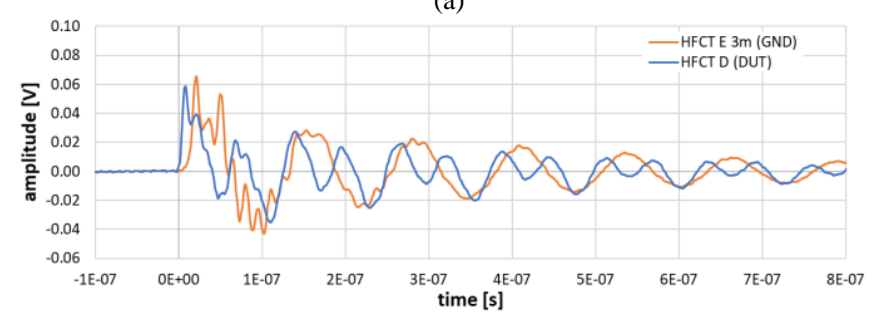

(b)

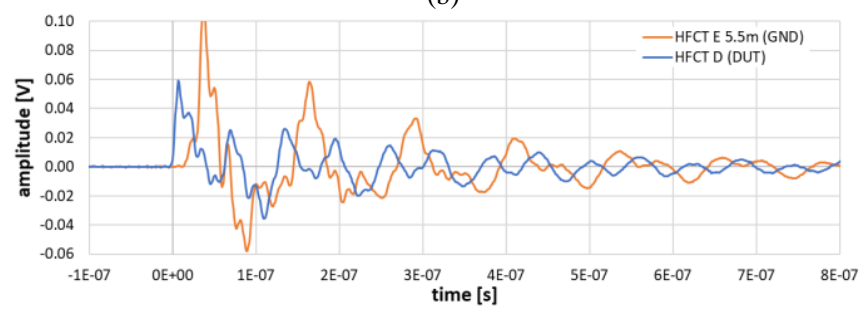

(c)

Fig. 16. Measured response of HFCT sensors in a conventional PD circuit to an injected $100 \mathrm{pC}$ pulse. (a) both sensors are located immediately after the test object.; (b) HFCT E is roughly midway along the ground conductor; (c) HFCT $\mathrm{E}$ is near the common ground point in the laboratory.

HFCT D was placed immediately after the test object $C_{\mathrm{a}}$ while HFCT E was displaced at intervals towards the common ground point $5.5 \mathrm{~m}$ from the test object. The test circuit was situated in a high voltage laboratory with "good" (low resistance) grounding. Nevertheless, for the frequency spectrum of the emitted signals, the laboratory ground appears to be an open connection or a high impedance. This impedance mismatch results in a discontinuity region at the ground interface which is seen as a near full reflection in Fig. 16c for HFCT E situated close to the ground common point. Time delay in the HFCT $\mathrm{E}$ signal is also consistent with propagation velocity along the bare ground conductor length $\left(5.5 \mathrm{~m}\right.$ at $3 \cdot 10^{8}$ $\mathrm{m} / \mathrm{s}$ gives $0.18 \cdot 10^{-7} \mathrm{~s}$ ). The midway point (Fig. 16b) shows a 
double peak caused by superposition of emitted signal from the test object and the reflected signal from the ground point. Almost identical signals are measured at the same location immediately after the test object $C_{\mathrm{a}}$ (Fig. 16a).

The same circuit was used to evaluate the applicability of the charge estimation techniques described in [26]. As expected for oscillatory signals, the resulting integrals are also oscillatory. Charge is be estimated from the integral's steady state value, but this is not clearly distinguishable from the measured data. Single integral values for charge shown in Table III are calculated from a restricted time period where oscillations at the peak of the integral give weakly steady-state average values prior to onset of decay. Setting integration limits $\left(t_{1}, t_{2}\right)$ manually for each measured signal is not practical as the response changes based on the sensor's interaction with the circuit and test object, and is open for interpretation. Methods to mitigate oscillations by means of low-pass filtering to facilitate this approach is discussed in [27]. Without any filtering or processing, HFCT $D$, which is placed in the immediate vicinity of the test object $C_{\mathrm{a}}$, approximates charge as roughly $92 \mathrm{pC}$ using the double integral approach for a $100 \mathrm{pC}$ injected pulse as shown in Fig. 17.

However, HFCT E, which is displaced along the length of the ground wire, is unable to converge to a value approximating the target charge of $100 \mathrm{pC}$. As evident in Figure 18, the single integral signal has returned to zero-level signifying peaking of the double integral for distances $3 \mathrm{~m}$ and $5.5 \mathrm{~m}$. Distance $0 \mathrm{~m}$ would require a longer integration time for HFCT $\mathrm{E}$ to determine a peak value using the double integral approach. Despite not providing a reliable charge estimation, the impact of sensor location is evident in Fig. 18 where charge calculations based on the peak of the double integral varies depending on the placement of the HFCT sensor along the ground wire. This once again emphasizes that, not only is the travelling wave phenomenon relevant within the test object itself, something as seemingly insignificant as placement of a HFCT sensor on a relatively short length of ground wire outside the test object contributes to the accuracy of PD assessment.

Neglecting the impact of the measurement circuit and travelling waves (measurement according to Fig. 9) does not improve the validity of the charge approximation methods for the developed HFCT sensors (Table IV). Figure 19 shows charge approximations for $100 \mathrm{pC}$ pulses measured by the HFCT sensors. Required integration times for the double integral method using the approximation $t=4 L_{2} / R$ far exceeds the measurement duration. Although not completely accurate (HFCT D peaks at $2.4 \mu$ s instead of the estimated $8.84 \mu$ s in Fig. 17), the approximation is indicative of the differences between the integration times of the constructed sensors as a function of their physical characteristics (inductance $L$ ) and can be used for design purposes. Longer acquisition times can extend evaluation periods, but as described earlier, this comes at the expense of pulse repetition rates. Furthermore, the compromise between record length and sampling rate is a considerable challenge and hardware restriction for performing pulse shape analysis together with charge estimation of PD events.

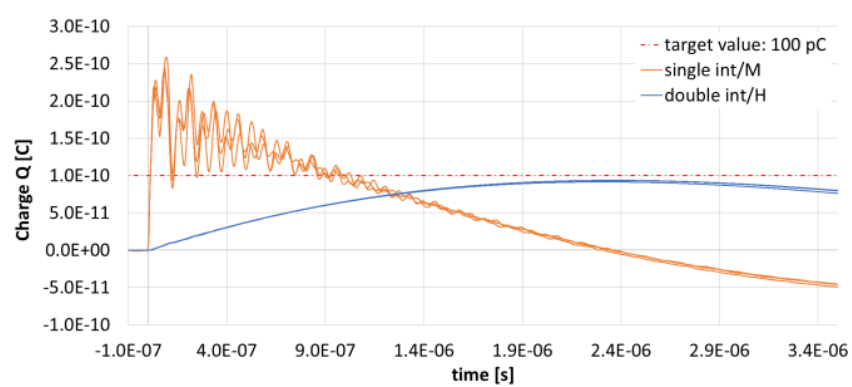

Fig. 17. Three measured signals by HFCT D observing $100 \mathrm{pC}$ injected into conventional PD circuit. The peak of the double integral divided by sensor gain $\mathrm{H}$ corresponds to the estimated charge value.

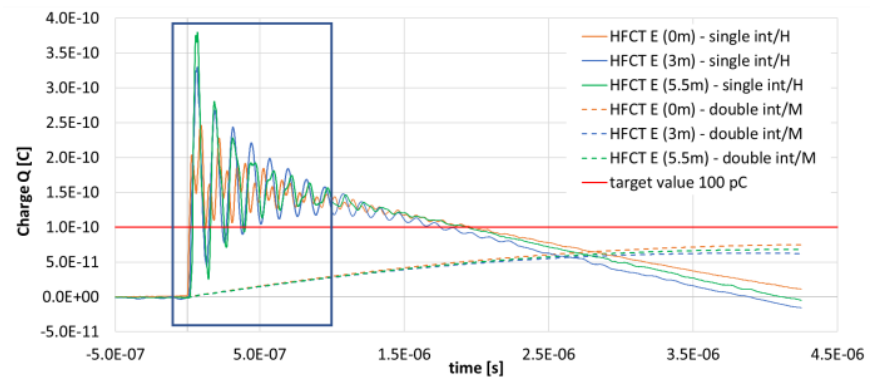

Fig. 18. Injected $100 \mathrm{pC}$ pulses measured by HFCT $\mathrm{E}$ at varying locations along ground wire. Integration period for the single integral method restricted to boxed area.

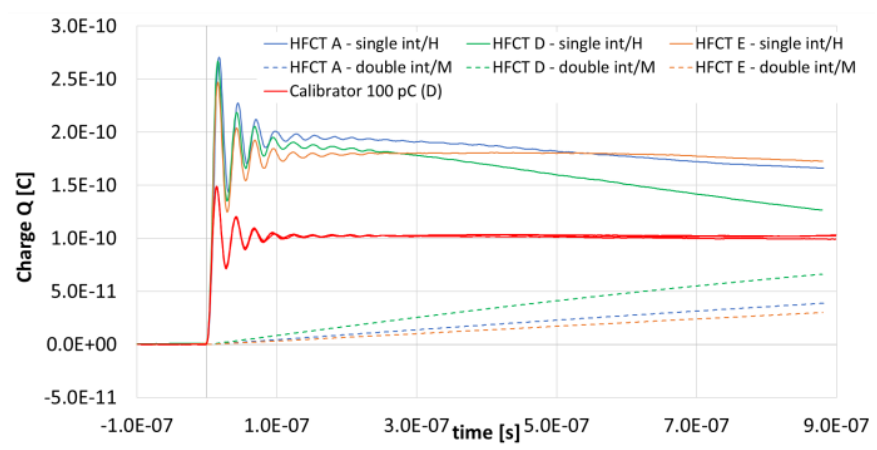

Fig. 19. Single and double integral methods for charge estimation of a $100 \mathrm{pC}$ calibrator pulse.

TABLE III

CHARGE ESTIMATION WITHIN CONVENTIONAL PD CIRCUIT

\begin{tabular}{lccccc}
\hline \hline & & \multicolumn{2}{c}{ HFCT D } & \multicolumn{2}{c}{ HFCT E } \\
\hline Charge estimation & Distance from & $q$ & $t_{1}-t_{2}$ & $q$ & $t_{1}-t_{2}$ \\
method: & $C_{\mathrm{a}}[\mathrm{m}]$ & {$[\mathrm{pC}]$} & {$[\mu \mathrm{s}]$} & {$[\mathrm{pC}]$} & {$[\mu \mathrm{s}]$} \\
& 0 & 129.6 & 0.75 & 142.1 & 0.95 \\
$q \approx \frac{1}{H} \int_{t_{2}}^{t_{1}} u(t) d t$ & 3 & 140.3 & 0.75 & 122.1 & 1.05 \\
& 5.5 & 136.9 & 0.75 & 115.9 & 0.95 \\
\hline Charge estimation & Distance from & $q$ & $4 L_{2} / R$ & $q$ & $4 L_{2} / R$ \\
method: & $C_{\mathrm{a}}[\mathrm{m}]$ & {$[\mathrm{pC}]$} & {$[\mu \mathrm{s}]$} & {$[\mathrm{pC}]$} & {$[\mu \mathrm{s}]$} \\
$q \approx \frac{1}{M} \iint_{0}^{4 L_{2} / R}$ & 0 & 93.5 & & 74.9 & \\
& 3 & 91.5 & 8.64 & 62.9 & 20.80 \\
\hline \hline
\end{tabular}

TABLE IV

CHARGE ESTIMATION WITHOUT PD CIRCUIT

\begin{tabular}{lccc}
\hline \hline Calibrator charge Q [pC] & 101.78 & 100.64 & 102.70 \\
Charge estimation method: & HFCT A & HFCT D & HFCT E \\
$q \approx \frac{1}{H} \int_{t_{2}}^{t_{1}} u(t) d t$ & 200.44 & 187.71 & 176.59 \\
$q \approx \frac{1}{M} \iint_{0}^{4 L_{2} / R} u(t) d t^{2}$ & 38.79 & 66.28 & 30.05 \\
Record length $[\mu \mathrm{s}]$ & 1.00 & 1.00 & 1.00 \\
Required integration time $[\mu \mathrm{s}]$ & 16.57 & 8.65 & 20.80 \\
\hline \hline
\end{tabular}




\section{CONCLUSIONS}

Despite the vast innovative work performed by all in this field, PD still largely remains a case-specific event as demonstrated in this paper. In particular, the concept of measurable "true" charge is illusive, and the validity distinction between calibration and normalization is complicated. Continued efforts are needed for better focused measurement procedures and sensor designs to enable unified practices and universal applications.

It is clear that high frequency signals attenuate rapidly with distance, and therefore sensors need to be close to the PD activity for adequate sensitivity. However, larger peak values can be observed farther away from the PD source as was demonstrated by constructive superposition of signals at the ground termination. This is not correlated to the physical event, but rather the local site characteristics and grounding practices.

Sensitivity of the constructed HFCTs without amplification is sufficient for detecting PD signals tens of $\mathrm{mV}$ in magnitude injected into a controlled laboratory test circuit with a lowcapacitance test object. Sensitivity needs to be improved for better low-level PD detection in more realistic application environments. Increasing gain by altering physical characteristics such as winding turns and core materials can migrate sensor characteristics away from the flat response desired for pulse shape analysis. For charge estimation, avoiding summation of multiple subsequent signals requires that integration times do not overlap with pulse repetition rates. However, interdependencies limit the design of sensors optimized for both accurate charge estimation and representative pulse shape analysis. The fundamental design question for PD applications using HFCTs is thus related to the intended primary purpose of the sensor. One may strive to combine both conventional coupling devices for charge estimation and wideband sensors for pulse shape assessment into the same circuit, but conventional devices load the circuit and thereby alter the waveform, whereupon HFCT placement becomes critical. If using multiple HFCT sensors distributed within the circuit, placement along grounding paths shall be similar (symmetrical) to allow for comparison.

\section{REFERENCES}

[1] J. V. Klüss, and A-P. Elg, "Challenges associated with implementation of HFCTs for partial discharge measurements," in Proc. CPEM 2020, Boulder, CO, USA, 2020.

[2] High-voltage test techniques - Partial discharge measurements, IEC 60270:2000+A1:2015 CSV Consolidated version, 2015.

[3] Rotating electrical machines - Part 27-2: On-line partial discharge measurements on the stator winding insulation of rotating electrical machines, IEC Technical Specification IEC/TS 60034-27-2, 2012.

[4] Electrical insulating materials and systems - Electrical measurement of partial discharges (PD) under short rise time and repetitive voltage impulses, IEC/TS 61934, 2011.

[5] Rotating electrical machines - Part 27-1: Off-line partial discharge measurements on the stator winding insulation of rotating electrical machines, IEC 60034-27-1, 2017.

[6] T. Klueter, J. Wulff and F. Jenau, "Time domain analysis of partial discharges at DC voltage in air and insulation oil," in Proc. EEEIC, Wroclaw, Poland, 2013, pp. 308-312.

[7] E. Lemke, "Using a field probe to study the mechanism of partial discharges in very small air gaps under direct voltage," IEEE Electr. Insul.
Mag., vol. 32, no. 4, pp. 43-51, Jul-Aug. 2016, 10.1109/MEI.2016.7528989

[8] X. Li, G. Wu, X. Zhang and S. Bian, "Partial discharge pulse shape detection and analysis under DC condition in typical defect models," in Proc. CEIDP, Vancouver, BC, Canada, 2007, pp. 188-191.

[9] A. Cavallini, G. C. Montanari, M. Tozzi and X. Chen, "Diagnostic of HVDC systems using partial discharges," in IEEE Trans. Dielectr. Electr. Insul., vol. 18, no. 1, pp. 275-284, Feb. 2011, 10.1109/TDEI.2011.5704519.

[10] S. Wenrong, L. Junhao, Y. Peng and L. Yanming, "Digital detection, grouping and classification of partial discharge signals at DC voltage," in IEEE Trans. Dielectr. Electr. Insul., vol. 15, no. 6, pp. 1663-1674, Dec. 2008, 10.1109/TDEI.2008.4712671.

[11] M. R. Rahimi, R. Javadinezhad and M. Vakilian, "DC partial discharge characteristics for corona, surface and void discharges," in Proc. ICPADM, Sydney, NSW, Australia, 2015, pp. 260-263.

[12] A. Pirker and U. Schichler, "Partial discharge measurement at DC voltage - Evaluation and characterization by NoDi* pattern," in IEEE Trans. Dielectr. Electr. Insul., vol. 25, no. 3, pp. 883-891, Jun. 2018, 10.1109/TDEI.2018.006742.

[13] F. P. Mohamed, W. H. Siew, J. J. Soraghan, S. M. Strachan and J. Mcwilliam, "The use of power frequency current transformers as partial discharge sensors for underground cables," in IEEE Trans. Dielectr. Electr. Insul., vol. 20, no. 3, pp. 814-824, Jun. 2013, 10.1109/TDEI.2013.6518951

[14] B. A. Siddiqui, P. Pakonen, P. Verho, "Novel sensor solutions for on-line PD monitoring," in Proc. CIRED, Lyon, France, Jun 2015.

[15] F. Alvarez, F. Garnacho, J. Ortego, M. A. Sanchez-Urán, "Application of HFCT and UHF sensors in on-line partial discharge measurements for insulation diagnosis of high-voltage equipment", in Sensors, vol. 15, no. 4, pp. 7360-7387, Apr. 2015, 10.3390/s150407360

[16] C. Zachariades, R. Shuttleworth, R. Giussani and R. MacKinlay, "Optimization of a High-Frequency Current Transformer Sensor for Partial Discharge Detection Using Finite-Element Analysis," in IEEE Sensors Journal, vol. 16, no. 20, pp. 7526-7533, Oct. 2016, 10.1109/JSEN.2016.2600272

[17] High voltage test techniques - Measurement of partial discharges by electromagnetic and acoustic methods, IEC TS 62478, 2016.

[18] P. Romano, G. Presti, A. Imburgia and R. Candela, "A new approach to partial discharge detection under DC voltage," in IEEE Electr. Insul. Mag., vol. 34, no. 4, pp. 32-41, Jul-Aug 2018, 10.1109/MEI.2018.8430041.

[19] P. H. F. Morshuis, "Time-resolved discharge measurements," in Proc. ICPD, Canterbury, UK, 1993, pp. 43-46.

[20] Xiaohua Li, Guangning Wu, Xueqin Zhang and Shanshan Bian, "Partial discharge pulse shape detection and analysis under DC condition," in Proc. EEIC, Nashville, TN, USA, 2007, pp. 48-51, 10.1109/EEIC.2007.4562586.

[21] Z. Chaojie, Y. Yi, Z. Ziyang and L. Yuncai, "Frequency domain analysis of electromagnetic wave radiated from partial discharge," in Proc. CMD, Bali, Indonesia, 2012, pp. 794-797, 10.1109/CMD.2012.6416267.

[22] G. Robles, J.M. Fresno, J.M. Martínez-Tarifa, J.A. Ardila-Rey, and E. Parrado-Hernández, "Partial Discharge Spectral Characterization in HF, VHF and UHF Bands Using Particle Swarm Optimization" in Sensors, vol. 18, no. 3, pp. 746, Mar. 2018, 10.3390/s18030746.

[23] A. Jaber, P. Lazaridis, Y. Zhang, D. Upton, H. Ahmed, U. Khan, B. Saeed, A. Mopty, P. Mather, M.F.Q. Vieira, R. Atkinson, M. Judd, and I.A. Glover, "Frequency Spectrum Analysis of Radiated Partial Discharge Signals", in Proc. IET EUROEM, London, UK, 2018.

[24] J. V. Kluss, M. R. Chalaki, and Z. Ahmed, "Influence of Sensor Selection for Observing Individual Partial Discharge Waveforms" in Proc. ISH, Budapest, Hungry, 2019, 10.1007/978-3-030-31680-8_67.

[25] F. Álvarez, F. Garnacho, A. Ramírez, E. Arcones, P. García, and C. A. Vera, "Generation of Reproducible Reference Insulation Defects in Experimental Tests Cells for Controlled PD monitoring," in Proc. ICHVE, Athens, Greece, 2018, pp. 1-4, 10.1109/ICHVE.2018.8641856.

[26] A. R. Mor, F. A. Munoz, and L. C. C. Heredia, "Principles of charge estimation methods using high-frequency current transformer sensors in partial discharge measurements", in Sensors, vol. 20 no. 9, pp. 2520 Apr. $2020,10.3390 / \mathrm{s} 20092520$

[27] A. R. Mor, L. C. C. Heredia and F. A. Muñoz, "Estimation of charge, energy and polarity of noisy partial discharge pulses," in IEEE Transactions on Dielectrics and Electrical Insulation, vol. 24, no. 4, pp. 2511-2521, 2017, 10.1109/TDEI.2017.006381. 